\title{
Silencing of vacuolar ATPase c subunit ATP6V0C inhibits the invasion of prostate cancer cells through a LASS2/TMSG1-independent manner
}

\author{
PENGCHENG ZOU ${ }^{1,2^{*}}$, YIFENG YANG $^{1 *}$, XIAOYAN XU ${ }^{1,3,4}$, BEIYING LIU ${ }^{5}$, \\ FANG MEI $^{1}$, JIANGFENG YOU ${ }^{1}$, QICHEN LIU ${ }^{6}$ and FEI PEI ${ }^{1}$ \\ ${ }^{1}$ Department of Pathology, School of Basic Medical Sciences, Peking University Health Science Center, \\ Beijing 100191; ${ }^{2}$ Department of Pathology, Qingdao Central Hospital, Qingdao 266000; \\ ${ }^{3}$ Department of Pathology, School of Basic Medical Sciences, Inner Monglia Medical College; \\ ${ }^{4}$ Department of Pathology, The Affiliated Hospital of Inner Monglia Medical College, Huhhot 010059; \\ ${ }^{5}$ School of Mechanical Engineering, University of Science and Technology Beijing, Beijing 100191; \\ ${ }^{6}$ Yuxin School of Capital Normal University, Beijing 100048, P.R. China
}

Received February 13, 2017; Accepted August 1, 2017

DOI: $10.3892 /$ or.2017.6092

\begin{abstract}
Vacuolar ATPase (V-ATPase), widespread in eukaryotic cells, is extensively expressed in many highly metastatic tumors, of which the V-ATPase c subunit ATP6V0C is particularly associated with the invasion and metastasis of cancer. ATP6V0C was directly found to interact with LASS2/TMSG1 which is a new tumor metastasis inhibitory gene identified by our laboratory in 1999. In order to study the role of ATP6V0C, we generated small interference RNA (siRNA) targeting ATP6V0C and investigated its function on the invasion of human prostate cancer cell line PC-3M-1E8 with high metastatic potential and its interplay with LASS2/TMSG1. We found that the expression of ATP6V0C was higher in prostate cancer cell lines PC-3M-1E8 and $\mathrm{PC}-3 \mathrm{M}$ with high metastatic potential than that from cell lines PC-3M-2B4 and PC-3 with low metastatic potential, indicating that ATP6V0C enhanced metastatic capacity in prostate cancer cells. Furthermore, silencing of ATP6V0C in PC-3M-1E8 cells inhibited V-ATPase activity (by $\sim 5$-fold), decreased extracellular hydrogen ion concentration and successively decreased activation of secreted MMP-9 (by $\sim 3$.6-fold), which coincided with the inhibition of cell migration and invasion in vitro, as well as a marked decrease in the expression of LASS2/TMSG1 probably through positive
\end{abstract}

Correspondence to: Dr Fei Pei, Department of Pathology, School of Basic Medical Sciences, Peking University Health Science Center, 38 Xue Yuan Road, Haidian, Beijing 100191, P.R. China

E-mail: peifei@bjmu.edu.cn

${ }^{*}$ Contributed equally

Key words: ATP6V0C, LASS2/TMSG1, prostate carcinoma, PC-3M-1E8 cells, siRNA, vacuolar $\mathrm{H}^{+}$ATPase feedback. Thus we concluded that silencing of the ATP6V0C gene effectively suppressed the migration and invasion of prostate carcinoma cells through the inhibition of the function of V-ATPase, not through a LASS2/TMSG1-dependent manner. Therefore ATP6V0C inhibitors are promising therapeutic targets for advanced prostate cancer.

\section{Introduction}

Prostate cancer represents the most common cancer and the second most common fatal cancer in men worldwide at present. Specifically, it is evaluated that prostate cancer accounts for $\sim 33 \%(233,000)$ of new cancer cases and 29,480 Americans may die from prostate cancer every year (1). The disease may progress from a hormone-sensitive to a castrate-resistant phenotype and eventually metastasize. Hence, further research is necessary to elucidate the molecular mechanism of prostate cancer (PCA) metastasis and explore the genes associated with PCA invasion and metastasis.

Vacuolar ATPase (V-ATPase), a multi-subunit enzyme, is an ATP-driven proton pump that translocates protons from the cytoplasm into intracellular compartments and across the plasma membrane and thus plays an important role in the formation and maintenance of the extracellular acidic microenvironment (2). V-ATPase consists of an ATP-hydrolyzing cytoplasmic V1 complex and a protontranslocation membrane-bound V0 complex. The V1 complex, which is composed of eight different subunits, hydrolyzes ATP to ADP to supply energy for $\mathrm{H}^{+}$transportation. The V0 complex, which is composed of five different subunits, forms the $\mathrm{H}^{+}$translocating channel. Of these subunits, subunit $\mathrm{c}$ in V0 (ATP6V0C) is a transmembrane proton transport channel, and in tumor cells it can pump protons out and cause extracellular acidification, which helps to maintain a relative basic cytoplasmic environment and a relative acidic extracellular environment. The low $\mathrm{pH}$ of the extracellular environment promotes the invasion and metastasis of cancer 
cells by activating proteolytic enzymes to degrade and remodel the extracellular matrix (ECM) $(3,4)$. Recently, ATP6V0C was revealed to directly bind to LASS2/TMSG1 $(5,6)$.

Homo sapien longevity assurance homolog 2 of yeast LAG1 (LASS2), which is also called tumor metastasis suppressor gene 1 (TMSG1; GenBank accession no. AF189062) or ceramide synthase 2 (CerS2), is a novel tumor-suppressor gene firstly identified from the human prostate cancer cell line PC-3M by our laboratory in 1999 and was designated as LASS2 in $2001(5,7,8)$. The LASS2/TMSG1 protein plays a key role in the inhibition of tumor invasion and metastasis probably through an ATP6V0C-dependent manner (9). However, the antitumor molecular mechanism of ATP6V0C in prostate cancer cells is unclear whether it is in a LASS2/TMSG1-dependent manner or not.

Therefore, in the present study, we utilized small interfering RNA (siRNA) technology to knockdown ATP6V0C expression in highly metastatic PC-3M-1E8 cells to further evaluate the effect of ATP6V0C on the migration and invasion of prostate cancer cells. Furthermore, we clarified the possible molecular mechanisms concerning the antitumor effect of ATP6V0C on the invasion of prostate cancer by detecting the activity of V-ATPase and the expression and activity of matrix metalloproteinase-2 (MMP-2) or MMP-9 and exploring its influence on the expression of mRNA and protein LASS2/TMSG1.

\section{Materials and methods}

Cell culture. Human prostate cancer cell line PC-3M (high metastatic variant) or PC-3M-1E8 cells (high metastatic cancer variant from human prostate cancer cell line $\mathrm{PC}-3 \mathrm{M}$; tumorigenicity frequency in nude mice, $100 \%$; spontaneous metastasis frequency in nude mice, 100\%) and PC-3 (low metastatic variant) or PC-3M-2B4 cells (non-metastatic cancer variant from human prostate carcinoma cell line PC-3M; tumorigenicity frequency in nude mice, $87.5 \%$; spontaneous metastasis frequency in nude mice, $0 \%$ ) were obtained from the Molecular Pathology Laboratory, Department of Pathology, Peking University Health Science Centre (7). The cells were maintained in RPMI-1640 medium supplemented with $10 \%$ fetal bovine serum (FBS) (HyClone, Logan, UT, USA) at $37^{\circ} \mathrm{C}$ with $5 \% \mathrm{CO}_{2}$.

RNA isolation, RT-PCR and real-time fluorogentic quantitative PCR (RFQ-PCR). Total cellular RNAs were isolated using the TRIzol reagent (Invitrogen, Carlsbad, CA, USA), and First-Strand cDNA synthesis was performed with $2 \mu \mathrm{g}$ total RNA and a reverse transcription system (TransGene, Beijing, China). Quantization of all gene transcripts was carried out by real-time quantitative PCR using TransStart Green qPCR SuperMix (TransGene) and an ABI Prism 7300 sequence detection system (Applied Biosystems, Foster City, CA, USA) with the expression of GAPDH as the internal control. The ATP6V0C or GADPH was amplified by RFQ-PCR under the following conditions: $95^{\circ} \mathrm{C}$ for $3 \mathrm{~min}$; followed by 45 cycles at $95^{\circ} \mathrm{C}$ for $30 \mathrm{sec}, 60^{\circ} \mathrm{C}$ for $30 \mathrm{sec}$, and $72^{\circ} \mathrm{C}$ for $30 \mathrm{sec}$. The primer pairs used were as follows: ATP6V0C forward, $5^{\prime}-\mathrm{ATG}$ TAAAGACCACCCCTCCT-3' and reverse, 5'-GAAACAGA CGATGGGCACTA-3'; LASS2/TMSG1 forward, 5'-CCTCTG ATGTCAAGCGAAAG-3' and reverse, 5'-GCAGGTAATCG
GAAGAGTCA-3'; GADPH forward, 5'-GGTCACCAGGGCT GCTTTTA-3' and reverse, 5'-TCCTGGAAGATGGTGAT GGG-3'. The experiment was repeated three times.

Western blot analysis. For immunoblotting, the cells were harvested and extracted in 4\% SDS buffer including protease inhibitor mixture (Roche, Mannheim, Germany) and centrifuged, and $10 \mu \mathrm{g}$ of the supernatant was subjected to SDS-PAGE using $10 \%$ polyacrylamide gels and transferred onto nitrocellulose membranes. The membranes were then incubated with primary antibodies: polyclonal goat anti-human-ATP6V0C (1:100), or polyclonal goat anti-human-LASS2/TMSG1 $(1: 2,000)$ (both from Santa Cruz Biotechnology, Santa Cruz, CA, USA) or polyclonal rabbit anti-human-MMP-2 $(1: 1,000)$ or polyclonal rabbit anti-human-MMP-9 antibodies (1:500) [both from Cell Signaling Technology (CST), Beverly, MA, USA], or monoclonal mouse anti-human- $\beta$-actin $(1: 2,500)$ overnight at $4^{\circ} \mathrm{C}$ followed by horseradish peroxidise-conjugated rabbit anti-goat secondary or goat anti-rabbit or goat anti-mouse secondary antibodies $(1: 5,000)$ (all from Santa Cruz Biotechnology). Immunodetection was performed using an enhanced chemiluminescence (ECL) system (Amersham Biosciences, Arlington Heights, IL, USA) according to the manufacturer's instructions.

RNA interference. siRNAs of ATP6V0C (GenBank accession no. NM001694) were synthesized by Qiagen Co. (Valencia, CA, USA). The sequences were as follows: nucleotides 693-713 of the human ATP6V0C cDNA (5'-CACAAAGTAGACCC TCTCCGA-3') were used as the target sequence of siRNA-1; nucleotides 715-735 of the human ATP6V0C cDNA (5'-CCC ACCAGCCACAGAATATTA-3') were used as the target sequence of siRNA-2; nucleotides $1139-1159$ of the human ATP6V0C cDNA (5'-TGCGCGGAGCTGTGTCCAATA-3') were used as the target sequence of siRNA-3; nucleotides 952-972 of the human ATP6V0C cDNA (5'-GCGGA TGATTTAGAATTGTCA-3') were used as the target sequence of siRNA-4. The non-specific control siRNA duplexes (AllStar negative control siRNA) were also purchased from the Qiagen Co. siRNAs were transfected into cells using Lipofectamine $^{\mathrm{TM}} 2000$ (Invitrogen) according to the manufacturer's instructions.

Activity of V-ATPase and extracellular $H^{+}$concentration. Assays of V-ATPase activity and extracellular $\mathrm{H}^{+}$concentration were performed as previously described by Xu et al (10).

Activity of MMP-2 and MMP-9 by gelatin zymography. An MMP zymography assay kit (Qiagen) was used to detect the activity of MMP-2 and MMP-9 according to the manufacturer's instructions.

Scratch-wound assay. Cells were harvested and suspended at $2.5 \times 10^{5}$ cells $/ \mathrm{ml}$ in RPMI-1640 medium. Subsequently, $2.5 \times 10^{5}$ cells in $1 \mathrm{ml}$ of medium were then cultured into a 6 -well plate. Forty-eight hours later, the cells were washed and supplemented with serum-free medium and fibronectin $(16 \mathrm{mg} / \mathrm{ml})$ for $24 \mathrm{~h}$. Confluent cell monolayers were scratched using a 200- $\mu 1$ Finnpipette ${ }^{\circledR}$ tip. The migration of cells into the wound was counted in multiple wells using a fluorescence 
microscope camera, and finally the rate of wound healing was calculated. The experiment was repeated three times.

Invasion assay. The Transwell invasion assays were performed using a Transwell chamber (BD Biosciences, Franklin Lakes, NJ, USA) with a Matrigel-coated filter. Cells $\left(2 \times 10^{5}\right)(400 \mu \mathrm{l})$ in exponential growth phase were placed on rehydrated Matrigel-coated culture inserts with $8-\mu \mathrm{m}$ diameter pore size membranes in 24-Transwell cell culture dishes and incubated for $12 \mathrm{~h}$. Subsequently, cells actively migrated from the upper to the lower side of the filter due to NIH3T3-conditioned serumfree medium which was used as an attractant. The cells on the upper side were removed using cotton swabs, and the invasive cells on the lower side were fixed, stained with hematoxylin and eosin (H\&E), and counted using a light microscope. The experiments were repeated three times.

Immunofluorescence by double staining. The variant-transfected PC-3M-1E8 cells were seeded on slides in RPMI-1640 medium containing 10\% FBS. Approximately $24 \mathrm{~h}$ after attachment, the cells were washed with phosphate-buffered saline (PBS), fixed in acetone for $30 \mathrm{~min}$, permeabilized with $0.1 \%$ (v/v) Triton X-100 in PBS, blocked with $1 \%$ BSA, and incubated with appropriate primary antibodies [goat polyclonal antibody against LASS2/TMSG1 (1:100) and rabbit polyclonal antibody against ATP6V0C (1:250)] followed by staining with secondary antibodies (Alex Fluor 488-conjugated rabbit anti-goat IgG (1:200 dilution) and Cy3-conjugated goat anti-rabbit IgG (1:200 dilution) (both from Invitrogen). The cells were washed four times and a final concentration of $0.1 \mathrm{~g} / \mathrm{ml} \mathrm{4,6-diamidino-2-phenylindole} \mathrm{(DAPI)} \mathrm{(Sigma,}$ St. Louis, MO, USA) was included in the last washing to stain the nuclei. Images were visualized and recorded using the Leica TCS SP5 fluorescence microscope (Leica Microsystems, Wetzlar, Germany).

Statistical analysis. Results are presented as the mean values and SEM. The data were subjected to Student's t-test (two-tailed; $\mathrm{P}<0.05$ was considered to indicate a statistically significant result).

\section{Results}

ATP6V0C expression in prostate cancer cell lines. Using semi-quantitative RT-PCR, the expression of ATP6V0C mRNA in the prostate cell lines PC-3M-1E8 $(0.370 \pm 0.023)$ and PC-3M $(0.195 \pm 0.011)$ with high metastatic potential was significantly higher than those in the cell lines PC-3M-2B4 $(0.054 \pm 0.009)$ and PC-3 $(0.091 \pm 0.005)$ with low metastatic potential $(\mathrm{P}<0.05$; Fig. 1A). By quantitative fluorescence real-time PCR method, the expression of ATP6V0C mRNA was higher in cell lines PC-3M-1E8 (1.000 \pm 0.000$)$ and PC-3M $(0.590 \pm 0.033)$ with high metastatic potential than that from cell lines PC-3M-2B4 $(0.146 \pm 0.024)$ and PC-3 $(0.191 \pm 0.010)$ with low metastatic potential, among which the expression of PC-3M-1E8 was the highest with statical significance $(\mathrm{P}<0.01$; $\mathrm{n}=3$; Fig. 1B).

Furthermore, the expression of the ATP6V0C protein in the four cell lines was detected using western blotting, which revealed that ATP6V0C was significantly higher in cell lines

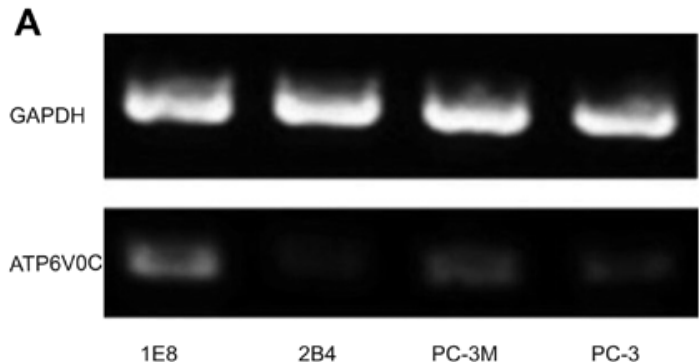

B

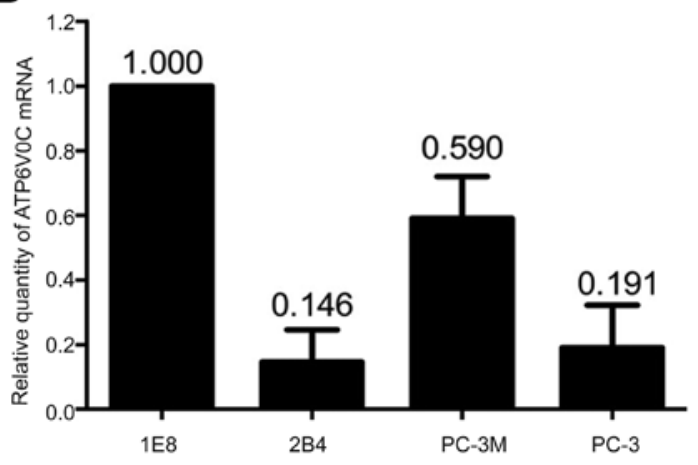

Figure 1. ATP6V0C mRNA expression in prostate cancer cell lines (A) Using semi-quantitative RT-PCR, the expression of ATP6V0C mRNA in the prostate cell lines PC-3M-1E8 $(0.370 \pm 0.023)$ and PC-3M $(0.195 \pm 0.011)$ with high metastatic potential was significantly higher than those in the cell lines PC-3M-2B4 (0.054 \pm 0.009$)$ and PC-3 (0.091 \pm 0.005$)$ with low metastatic potential $(\mathrm{P}<0.05)$. (B) By quantitative fluorescence real-time PCR method, the expression of ATP6V0C mRNA was higher in cell lines PC-3M-1E8 $(1.000 \pm 0.000)$ and $\mathrm{PC}-3 \mathrm{M}(0.590 \pm 0.033)$ with high metastatic potential than that from cell lines PC-3M-2B4 $(0.146 \pm 0.024)$ and PC-3 $(0.191 \pm 0.010)$ with low metastatic potential $(\mathrm{P}<0.01 ; \mathrm{n}=3)$.

PC-3M-1E8 $(0.481 \pm 0.090)$ and PC-3M $(0.333 \pm 0.080)$ with high metastatic potential than that from cell lines $\mathrm{PC}-3 \mathrm{M}-2 \mathrm{~B} 4$ $(0.115 \pm 0.030)$ and PC-3 $(0.128 \pm 0.040)$ with low metastatic potential ( $\mathrm{P}=0.002$; Fig. $2 \mathrm{~A}$ and $\mathrm{B})$.

Therefore, $\mathrm{PC}-3 \mathrm{M}-1 \mathrm{E} 8$ cells that had the highest expression of ATP6V0C were chosen for the gene silencing experiment.

Effects of ATP6VOC siRNAs on the expression of ATP6V0C in PC-3M-1E8 cells. We examined four ATP6V0C siRNAs to target human ATP6V0C as aforementioned. RFQ-PCR revealed a marked decrease of $87 \%$ with siRNA-3, $71.2 \%$ with siRNA-1 and $61.7 \%$ with siRNA-2 in the levels of ATP6V0C mRNA after transfection with siRNAs in PC-3M-1E8 cells, compared with the ATP6V0C AllStar negative control siRNA (unrelated negative control group) or the untransfected cultures (control group) $(\mathrm{P}<0.05$; Fig. 3A). However, there was no statistical significance between the unrelated negative control and control group. Moreover, we found the expression of ATP6V0C mRNA in cells transfected with siRNA-3, siRNA-1 and siRNA-2 all decreased to varying degrees as determined by RT-PCR, among which the interference of siRNA-3 was the most significant and consistent with the results of real-time PCR (Fig. 3B).

Moreover, the expression of the ATP6V0C protein was detected by western blotting before and after silencing, and the results revealed that ATP6V0C siRNA-3 suppressed ATP6V0C expression to $46.5 \%$ of that in the control cultures $(\mathrm{P}<0.01$; Fig. 4A and B). 

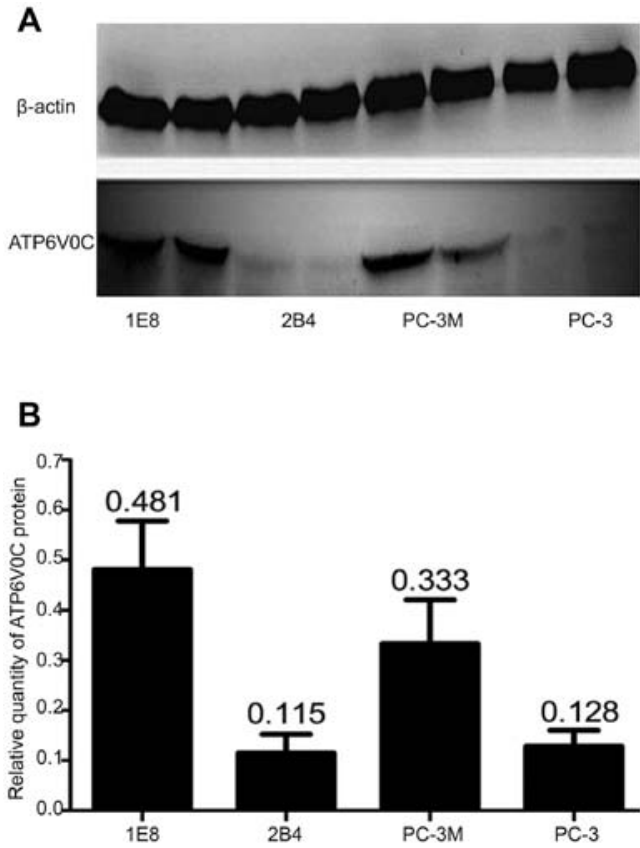

Figure 2. ATP6V0C protein expression in prostate cancer cell lines. (A and B) The expression of the ATP6V0C protein in the four cell lines was detected by western blotting, which revealed that ATP6V0C is significantly higher in cell lines PC-3M-1E8 $(0.481 \pm 0.090)$ and PC-3M $(0.333 \pm 0.080)$ with high metastatic potential than that from cell lines PC-3M-2B4 $(0.115 \pm 0.030)$ and PC-3 $(0.128 \pm 0.040)$ with low metastatic potential $(\mathrm{P}=0.002)$.

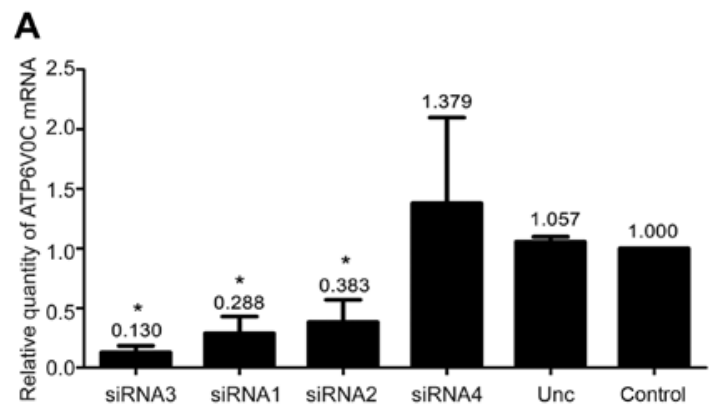

B
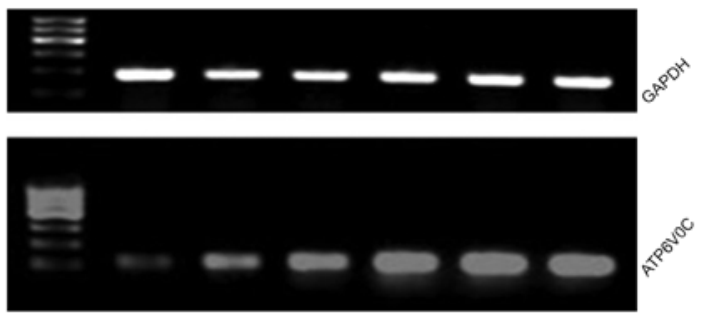

Marker siRNA3 siRNA1 SiRNA2 siRNA4 Unc Control

Figure 3. Effects of ATP6V0C siRNAs on the expression of ATP6V0C in PC-3M-1E8 cells. (A) RFQ-PCR revealed markedly reduction of $87 \%$ with siRNA-3, $71.2 \%$ with siRNA-1, and $61.7 \%$ with siRNA-2 in the levels of ATP6V0C mRNA after transfection with siRNAs in PC-3M-1E8 cells, compared with the ATP6V0C AllStar negative control siRNA or the untransfected cultures ( $\mathrm{P}<0.05$ ). (B) The expression of ATP6V0C mRNA in cells transfected with siRNA-3, siRNA-1 and siRNA-2 all decreased to varying degrees as determined by RT-PCR, among which the interference of siRNA-3 was the most significant.

Hence, we selected siRNA-3 as an effective siRNA for subsequent experiments.

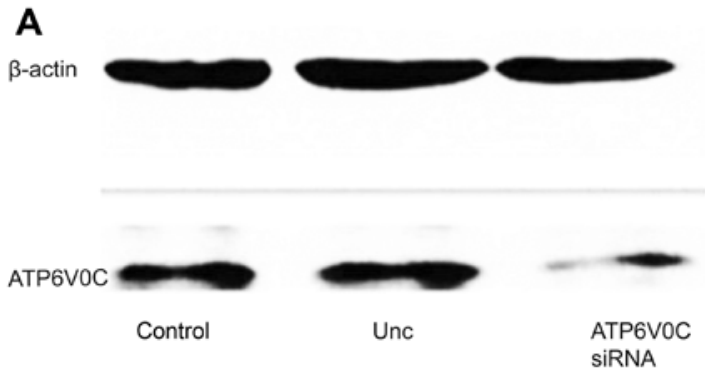

B

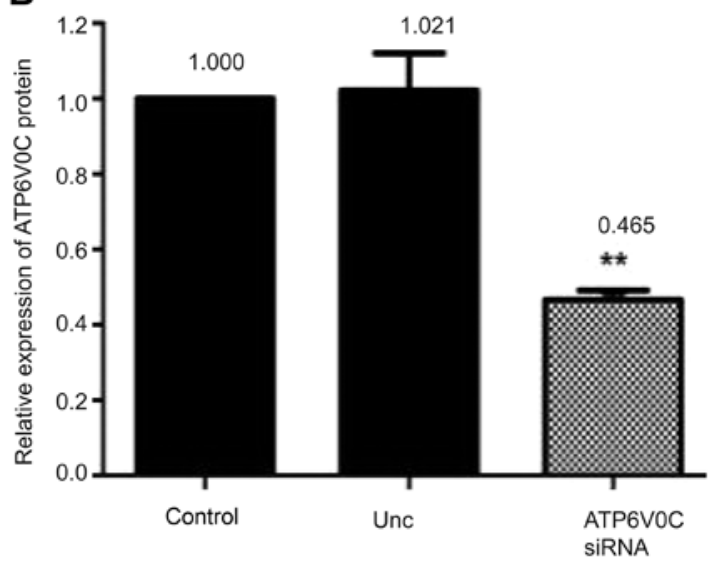

Figure 4. Effects of ATP6V0C-siRNA on the expression of ATP6V0C in PC-3M-1E8 cells. (A and B) The expression of the ATP6V0C protein was detected by western blotting before and after interference, which revealed that ATP6V0C-siRNA-3 suppressed ATP6V0C production to $46.5 \%$ of that in the control cultures $\left({ }^{* *} \mathrm{P}<0.01\right)$.

ATP6VOC-siRNA decreases the activity of V-ATPase in $P C-3 M-1 E 8$ cells. The activity of V-ATPase was detected by the Gemend's V-ATPase activity assay kit (Gemend Scientifics, Inc.). The activity of V-ATPase = OD of sample/ (concentration $\times 31.1) \times 10^{3} \mathrm{U} / \mathrm{mg}$. The activity of V-ATPase was $6.867 \pm 0.306$ in the control, $6.583 \pm 0.705$ in the unrelated negative control group, and $1.297 \pm 0.342$ in the ATP6V0CsiRNA transfected cells, indicating that ATP6V0C-siRNA decreased the V-ATPase activity of cells markedly (-5-fold; $\mathrm{P}<0.01 ; \mathrm{n}=3$ ), as shown in Fig. 5A.

ATP6VOC-siRNA decreases extracellular $\mathrm{H}^{+}$concentration in $P C-3 M-1 E 8$ cells. Extracellular $\mathrm{H}^{+}$concentration was detected by $\mathrm{pH}$-sensitive fluorescence probe bis-carboxy-ethylcarboxy-fluorescein (BCECF), as shown in Fig. 5B. The proton secretion of the ATP6V0C-siRNA cells was notably decreased at 12 and $18 \mathrm{~h}$ compared with that of the controlsiRNA cells or the untransfected cells $(\mathrm{P}<0.01 ; \mathrm{n}=3$, Fig. 5B).

ATP6VOC-siRNA inhibits the activity of MMP-9 in the supernatant of the culture cells. We studied the expression, secretion and activity of MMP-2 and MMP-9 in the three groups of cells, which were closely relative to cancer metastasis on the basis of a previous study (10). The results revealed that there was no significant difference in the expression and secretion of MMP-2 (Fig. 6A) and MMP-9 (Fig. 6B) proteins among the ATP6V0C-siRNA, control-siRNA or untreated cells in total protein and cell supernatants of the PC-3M-1E8 cells, indicating that the 
A

\begin{tabular}{lcccc}
\hline \multirow{2}{*}{ Cell lines } & \multicolumn{3}{c}{ V-ATPase activity } & $\bar{X} \pm$ SD \\
\cline { 2 - 4 } & First & Second & Third & \\
\hline Control & 6.60 & 7.20 & 6.80 & $6.867 \pm 0.306$ \\
Unc & 5.77 & 7.02 & 6.96 & $6.583 \pm 0.705$ \\
ATP6V0C siRNA & 0.98 & 1.66 & 1.25 & $1.297 \pm 0.342$ \\
\hline
\end{tabular}

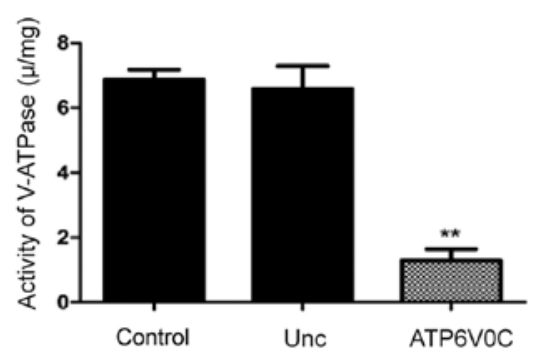

B

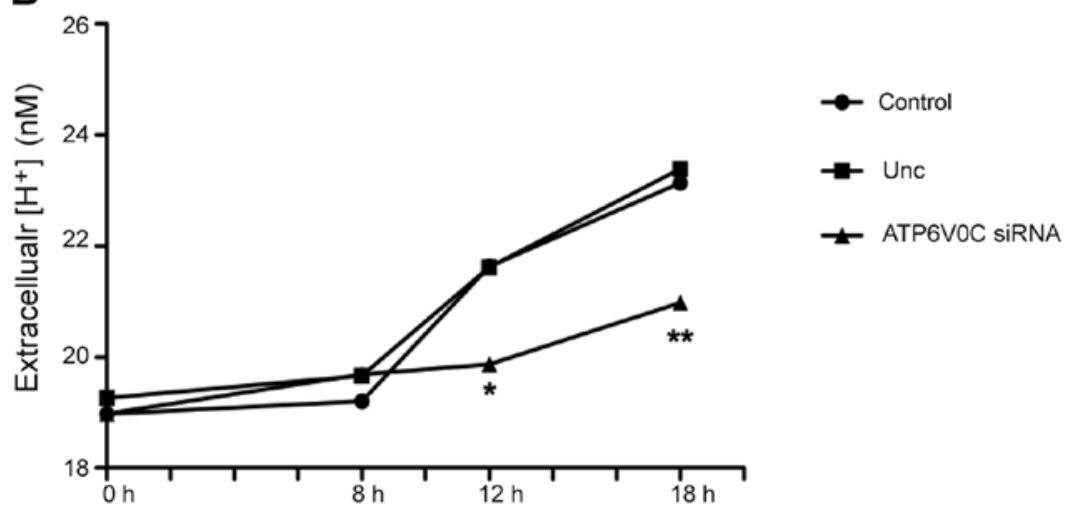

Figure 5. ATP6V0C-siRNA decreases the activity of V-ATPase and extracellular $\mathrm{H}^{+}$concentration in PC-3M-1E8 cells. (A) The activity of V-ATPase was $6.867 \pm 0.306$ in the control, $6.583 \pm 0.705$ in the unrelated negative control group, and $1.297 \pm 0.342$ in the ATP6V0C-siRNA transfected cells, revealing that the V-ATPase activity in ATP6V0C-siRNA cells was markedly decreased when compared to the control and the unrelated negative group $\left({ }^{* *} \mathrm{P}<0.01 ; \mathrm{n}=3\right)$. (B) Extracellular $\mathrm{H}^{+}$concentration was detected by $\mathrm{pH}$-sensitive fluorescence probe bis-carboxy-ethyl-carboxy-fluorescein (BCECF). The proton secretion of the ATP6V0C-siRNA cells was notably decreased at 12 and $18 \mathrm{~h}$ compared with that of the control-siRNA cells or untransfected cells ( $\mathrm{P}<0.01 ; \mathrm{n}=3$ ).

A

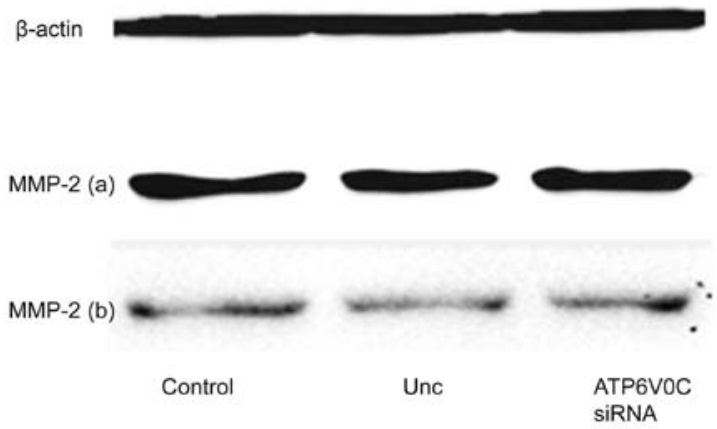

B

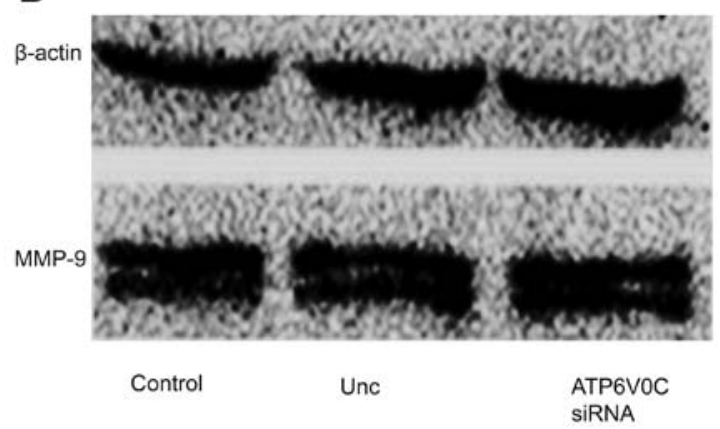

Figure 6. Effect of ATP6V0C-siRNA on the expression and secretion of MMP-2 and MMP-9 in the culture cells. (A) Western blotting revealed that there was no significant difference in the (a) expression and (b) secretion of the MMP-2 protein among the ATP6V0C-siRNA, control-siRNA or untransfected cells in total protein and cell supernatants of the PC-3M-1E8 cells. (B) Western blotting revealed that there was no significant difference in the expression of the MMP-9 protein among the three groups (the secretion of MMP-9 was not possibly detected due to a very low concentration). decrease of ATP6V0C expression had no effect on the expression and secretion of MMP-2 and MMP-9 proteins (the secretion of MMP-9 was not detected possibly due to a very low concentration).

Moreover, the gelatinase activity assay indicated that the activity of secreted MMP-9 is $46.62 \%$ in control and $41.98 \%$ in unrelated negative control group, and $11.40 \%$ in ATP6V0C siRNA transfected cells (Fig. 7A and B), indicating that the downregulation of ATP6V0C decreased the activity of secreted MMP-9 (by $~ 3.6$-fold) (the activity of MMP-2 had not been detected).

siRNA targeting ATP6VOC weakens the migration and invasion of PC-3M-1E8 cells. In the wound migration assay, the cell-free wound gaps in the ATP6V0C-siRNA transfected cells healed slowler than that in the untreated cells or the AllStar negative control siRNA-transfected cells (Fig. 8A). The migration rate in the untreated cells, the AllStar negative control siRNA-transfected and the ATP6V0C-siRNA transfected cells at $24 \mathrm{~h}$ after wounding was $47 \pm 1.00,50.67 \pm 1.45$ and $22.33 \pm 1.20 \%$, respectively, with statistical significance $(\mathrm{P}<0.01)$.

However, using the Boyden chamber invasion assay, the ATP6V0C-siRNA transfected cells revealed a markedly decreased invasion ability when compared with the control cells (Fig. 8B). The number of invasive cells in the untreated group, the AllStar negative control siRNA-transfected and the ATP6V0C-siRNA transfected groups was $67.67 \pm 2.186$, $71.00 \pm 3.055$ and $38.33 \pm 1.202$, respectively. The difference was statistically significant $(\mathrm{P}<0.01)$. 
A

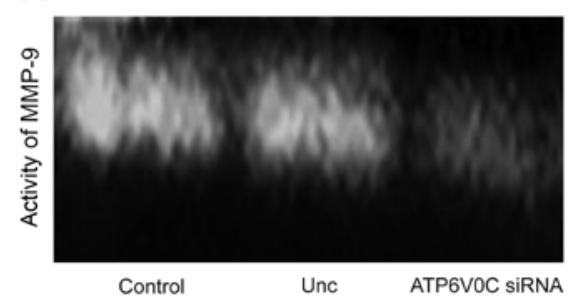

B

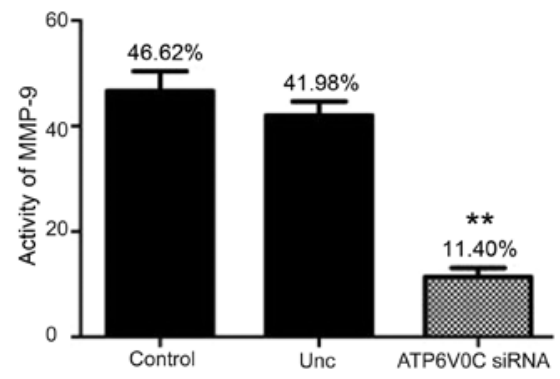

Figure 7. Effect of ATP6VOC-siRNA on the activity of MMP-9 in the cultured cells. (A and B) The supernatant of cultured cells was collected and the gelatinase activity was assayed with gelatin zymography. The activity of secreted MMP-9 was $46.62 \%$ in the control, $41.98 \%$ in the unrelated negative control group, and $11.40 \%$ in the ATP6V0C-siRNA transfected cells, indicating that the downregulation of ATP6V0C decreased the activity of secreted MMP-9 (by $\sim 3.6$-fold). ${ }^{* *} \mathrm{P}<0.01$. (The activity of MMP-2 was not detected).

ATP6VOC-siRNA inhibits the expression of LASS2/TMSG1 in PC-3M-1E8 cells. Using semi-quantitative RT-PCR, the expression of LASS2/TMSG1 mRNA in the ATP6V0C-siRNA cells decreased by 77 and $74 \%$ compared to the control cells and the Unc group, respectively ( $\mathrm{P}<0.01$; Fig. 9A and B). Real-time
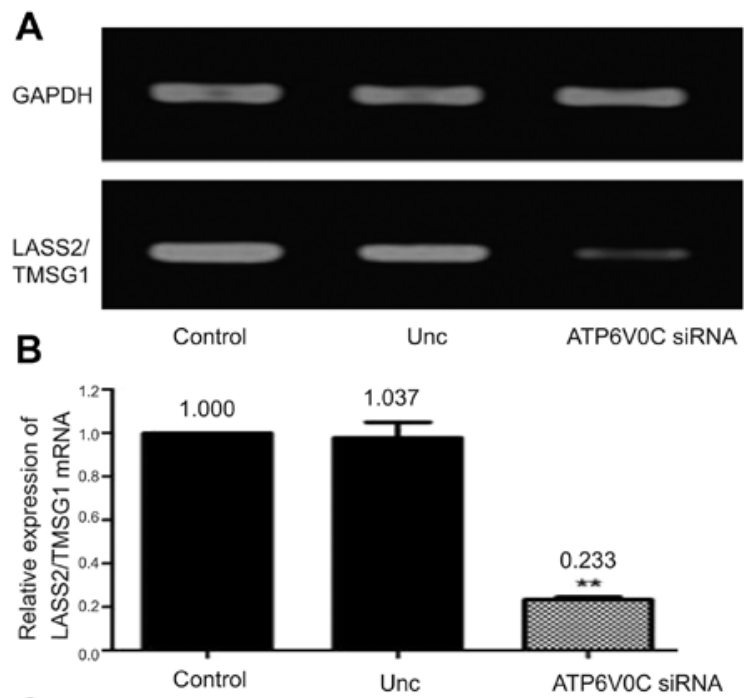

C

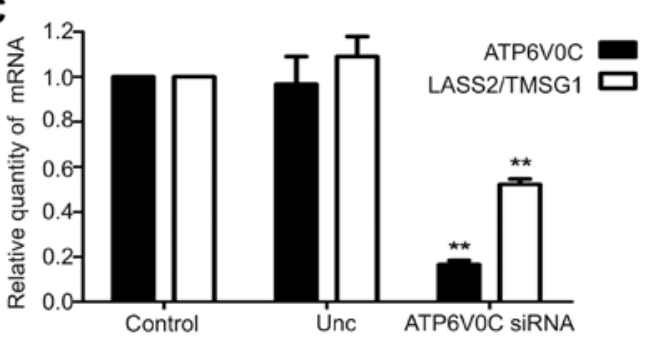

Figure 9. ATP6V0C-siRNA inhibits the expression of LASS2/TMSG1 in PC-3M-1E8 cells. (A and B) Using semi-quantitative RT-PCR, the expression of LASS2/TMSG1 mRNA in the ATP6V0C-siRNA cells was decreased by 77 and $74 \%$ compared to the control cells and the Unc group, respectively $\left({ }^{* *} \mathrm{P}<0.01\right)$. (C) Real-time PCR also provided the same result. When the ATP6V0C gene was interfered with, the expression of LASS2/TMSG1 mRNA was markedly inhibited compared to the control cells and the Unc group $\left({ }^{* *} \mathrm{P}<0.01\right)$.

A

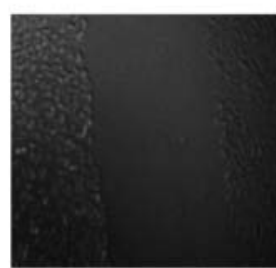

Cell scratch $(\mathrm{O} h)$

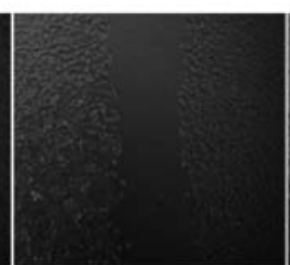

Control (24 h)

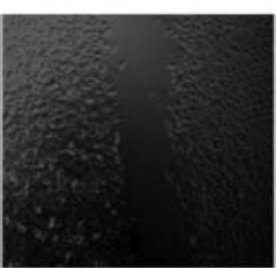

Unc (24 h)

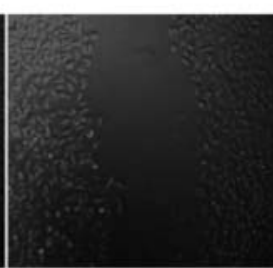

ATP6VOC siRNA (24 h)

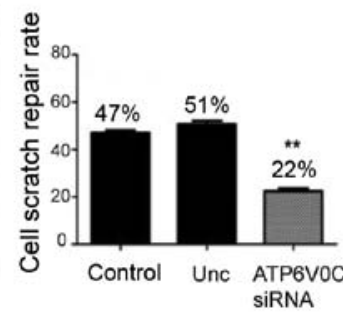

B
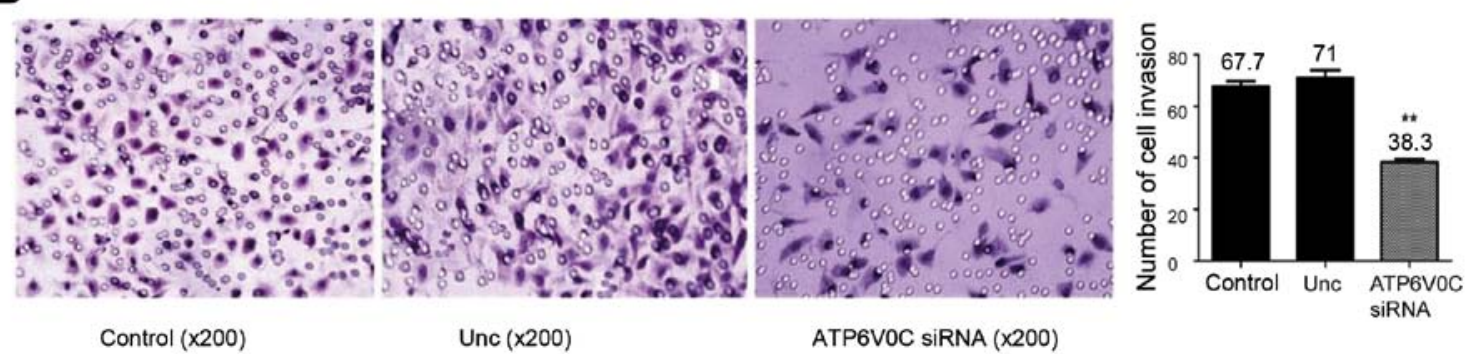

Figure 8. siRNA targeting ATP6V0C weakens the migration and invasion of PC-3M-1E8 cells. (A) In the wound migration assay, the cell-free wound gaps in the ATP6V0C-siRNA transfected cells healed slowler than that in the untreated cells or the AllStar negative control siRNA-transfected cells. The migration rate in the untreated cells, the AllStar negative control siRNA-transfected and the ATP6V0C-siRNA transfected cells at $24 \mathrm{~h}$ after wounding was $47 \pm 1.00$, $50.67 \pm 1.45$ and $22.33 \pm 1.20 \%$, respectively, with statistical significance $\left.{ }^{* *} \mathrm{P}<0.01\right)$. (B) Using a Boyden chamber invasion assay, the ATP6V0C-siRNA transfected cells displayed a markedly decreased invasion ability when compared with the untreated cells or the AllStar negative control siRNA-transfected cells. The number of invasive cells in the untreated group, the AllStar negative control siRNA-transfected group and the ATP6V0C-siRNA transfected group was $67.67 \pm 2.186,71.00 \pm 3.055$ and $38.33 \pm 1.202$, respectively. The difference was statistically significant $\left({ }^{* *} \mathrm{P}<0.01\right)$. 
A $\beta$-actin

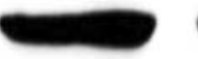
LASS2/
TMSG1
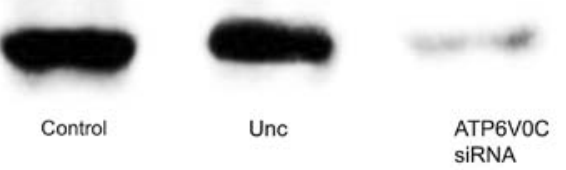

B

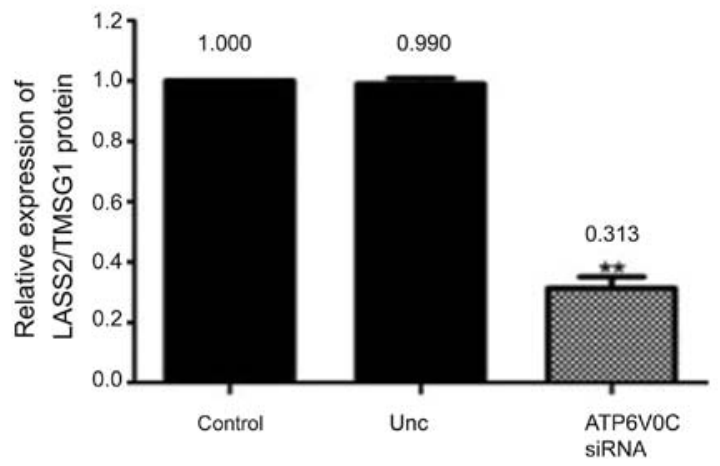

Figure 10. ATP6V0C-siRNA inhibits the expression of LASS2/TMSG1 in PC-3M-1E8 cells. (A and B) The expression of the ATP6V0C protein in the three cell lines was detected by western blotting, which revealed that the expression of the LASS2/TMSG1 protein in the ATP6V0C-siRNA group was decreased by 68.7 and $67.7 \%$ compared to the control and the Unc group, respectively $\left({ }^{* *} \mathrm{P}<0.01\right)$.
PCR also provided the same result. When the ATP6V0C gene was interfered with, the expression of LASS2/TMSG1 mRNA was markedly inhibited compared to the control cells and the Unc group ( $\mathrm{P}<0.01$; Fig. 9C).

Moreover, the expression of the ATP6V0C protein in the three cell lines was detected by western blotting, which revealed that the expression of the LASS2/TMSG1 protein in the ATP6V0C-siRNA group was decreased by 68.7 and $67.7 \%$ compared to the control and the Unc group, respectively $(\mathrm{P}<0.01$; Fig. $10 \mathrm{~A}$ and $\mathrm{B})$.

Furthermore, double immunofluorescence staining confirmed the interaction between ATP6V0C and LASS2/TMSG1, revealing that the LASS2/TMSG1 protein (green) and the ATP6V0C protein (red) were mainly co-localized in the plasma (yellow). After the PC-3M-1E8 cells were transfected with siRNA-ATP6V0C, the expression of LASS2/TMSG1 and ATP6V0C were both significantly decreased compared to the control group and the negative group, and the co-localization signal was markedly decreased (Fig. 11).

\section{Discussion}

At present, it has been found that ATP6V0C is expressed in many tumors, particularly tumors with high metastatic potential. It has been reported that the ATP6V0C protein was mildly
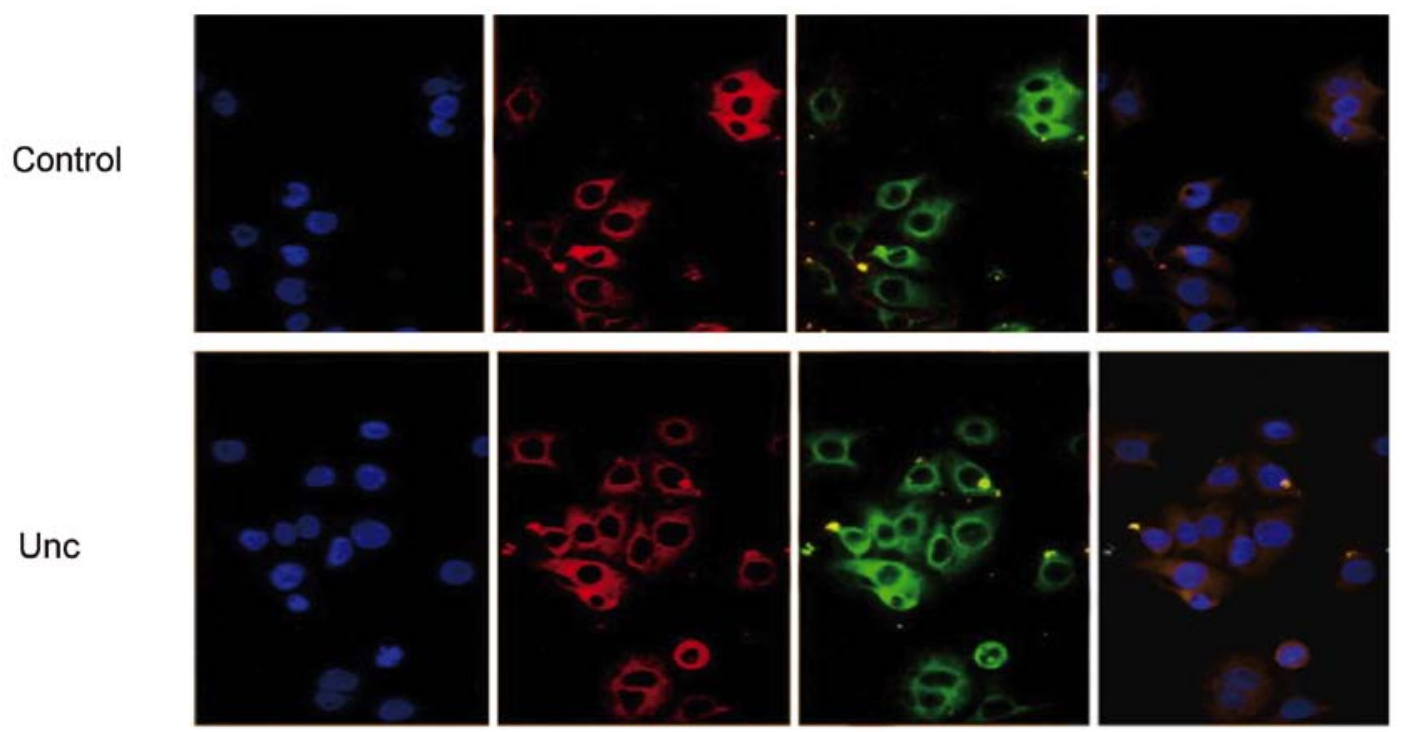

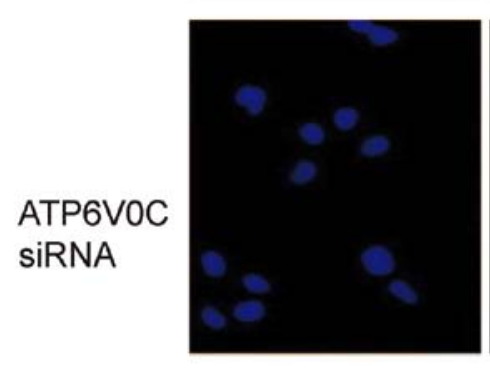

DAPI

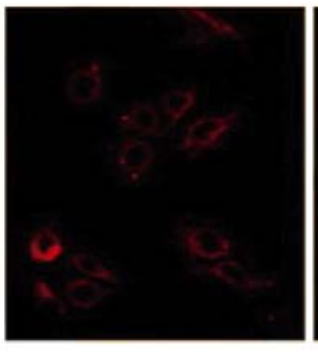

Anti-ATP6VOC

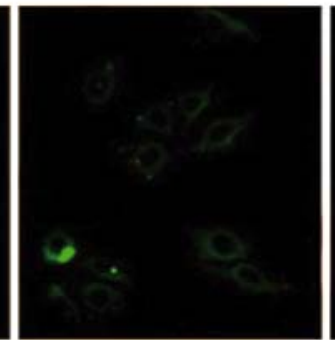

Anti-LASS2/TMSG1

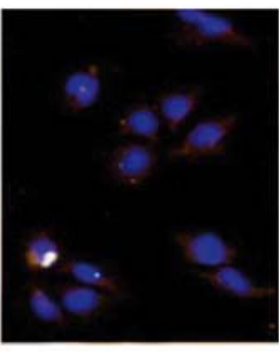

Merged

Figure 11. Double immunofluorescence staining confirmed the interaction between ATP6V0C and LASS2/TMSG1, revealing that the LASS2/TMSG1 protein (green) and the ATP6V0C protein (red) were mainly co-localized in the plasma (yellow). After the PC-3M-1E8 cells were transfected with siRNA-ATP6V0C, the expression of both LASS2/TMSG1 and ATP6V0C was significantly decreased compared to the control and the negative group, and the co-localization signal was markedly decreased. 
to markedly expressed in invasive pancreatic cancer (42 out of 46, 92\%), but was not detected in non-invasive pancreatic cancer $(11,12)$. As for breast cancer, ATP6V0C was heavily expressed in tumors with high metastatic potential while it was poorly expressed in those with low metastatic potential (13). However, in prostate cancer the expression of ATP6V0C was unknown.

In the present study, we detected the expression of ATP6V0C in prostate cancer cell lines with different metastatic potential using various methods such as semi-quantitative RT-PCR, quantitative fluorescence real-time PCR and western blotting and found that the expression of ATP6V0C was both higher in high metastatic potential cell lines PC-3M-1E8 and PC-3M than that in low metastatic potential cell lines PC-3M-2B4 and PC-3, among which the expression peak was observed in the PC-3M-1E8 cells, indicating a strong positive relationship between ATP6V0C expression and tumor progression. It has been demonstrated that ATP6V0C is a tumor metastasis-related gene and plays a key role in the invasion and metastasis of tumors. Thus, we chose PC-3M-1E8 cells for ATP6V0C gene silencing.

Subsequently, we designed and synthesized ATP6V0CsiRNA to downregulate the expression of ATP6V0C in the human prostate cancer cell line PC-3M-1E8 with high metastatic potential, hoping to explore the effect and molecular mechanism of ATP6V0C on tumor migration and invasion in prostate cancer. In addition, we established two control groups, one in which the control group was the untransfected cultures, and the other the unrelated negative control group was the non-specific control siRNA-duplexes (AllStar negative control siRNA) transfected cultures, and found that there was no statistical significance between the two control groups. Furthermore, we found the activity of vacuolar ATPase (V-ATPase) in tumor cells significantly decreased after transfection with specific siRNA of ATP6V0C as determined using a V-ATPase activity detection kit. Furthermore, with the use of BCECF proton sensitive fluorescence probe detection, we found that the concentration of protons outside the transfected cells was significantly decreased. The results revealed that silencing of ATP6V0C inhibited the activity of V-ATPase, and in turn inhibited the function of the transmembrane proton pump and as a result less protons were pumped outside of the cell thus leading to a decrease in extracellular $\mathrm{H}^{+}$concentration in cancer cells.

Moreover, we used a zymography kit to detect the activity of MMP-2 and MMP-9, and found that the activity of MMP-9 was significantly decreased in the ATP6V0C-siRNA cell supernatant, possibly due to the low extracellular $\left(\mathrm{H}^{+}\right)$ concentration which inhibited the activation of proton sensitive proteolytic enzymes, such as the MMP family (including MMP-9) (14). The decreased activity of MMP-9 impeded degradation and remodeling of ECM, therefore inhibiting the migration and invasion of tumor cells. The scratch repair experiments and Transwell invasion experiment in vitro both demonstrated that, after silencing of ATP6V0C expression, the ability of migration and invasion of PC-3M-1E8 cells was significantly decreased and it was consistent with previous studies in which bafilomycin A1 (proton pump inhibitor) suppressed cell motility (15). In addition, ATP6V0C itself has binding sites for the papillomavirus E5 oncoprotein, the platelet-derived growth factor $\beta$ receptor and $\beta 1$ integrin, which can also regulate proliferation and adhesion (15).

Moreover, we ascertained whether ATP6V0C silencing, which inhibited the ability of migration and invasion of PC-3M-1E8 cells, was associated with LASS2/TMSG1. LASS2/TMSG1 is a new tumor metastasis suppressor gene which was first discovered by our laboratory in 1999 (16). In 2002, Pan et al found that the LASS2/TMSG1 protein could bind directly with ATP6V0C and inhibit the activity of V-ATPase, thus inhibiting the growth, infiltration and metastasis of tumors as determined by yeast two hybrid and GST pull-down experiments. In 2012, Fan et al found that LASS2/TMSG1 enhanced the chemosensitivity in cancer cells by inhibiting the V-ATPase activity through binding to ATP6V0C (17). In 2014, Xu et al (from our laboratory) confirmed that decreasing the expression of LASS2/TMSG1 by gene interference could increase the proton concentration outside the cell, enhance the activity of V-ATPase, while also inhibit apoptosis of tumor cells and promote invasion or metastasis of prostate cancer $(10,18,19)$. In 2015 , Mei et al found that LASS2/TMSG1 inhibited the growth and invasion of breast cancer cells in vitro through regulation of V-ATPase activity (20). A great amount of research has focused on the influence of LASS2/TMSG1 on ATP6V0C, however, does ATP6V0C have feedback regulation on LASS2/TMSG1 expression? To investigate this, in the present study, we used siRNA to interfere with the expression of ATP6V0C in high metastatic human prostate cancer cell line PC-3M-1E8 and to study its influence on the expression of LASS2/TMSG1 mRNA and protein, RT-PCR, real-time PCR and western blotting were used, demonstrating that after silencing of ATP6V0C, the expression of LASS2/TMSG1 was significantly less than that in the control and negative group. Then, we used an immunofluorescence double staining technique to directly observe the interaction between ATP6V0C and LASS2/TMSG1, and found that the expression and co-localization signal of these two proteins both weakened significantly after interference, suggesting that ATP6V0C may have feedback regulation on LASS2/TMSG1 expression and silencing of ATP6V0C may inhibit the migration and invasion of prostate cancer cells through a LASS2/TMSG1-independent manner.

However, the molecular mechanism of ATP6V0C-positive regulation of the expression of LASS2/TMSG1 is unclear. In 2011, Gong et al (of our laboratory) identified a potential enhancer and three potential silencers in the 5'-flanking region of LASS2/TMSG1, and demonstrated that transcription factor KLF6 and Sp1 had a combined effect on the regulatory sequence in the first exon of the LASS2/TMSG1 gene as deterimined through protein immunoprecipitation, chromatin immunoprecipitation and gel mobility shift experiments, thereafter initiating the transcriptional activation of LASS2/TMSG1 in prostate cancer cells (21). Furthermore, more than one inhibitory regulatory region upstream of the transcriptional initial point of the LASS2/TMSG1 gene was discovered. In 2015, Fan et al found that the expression of LASS2/TMSG1 was transcriptionally activated by KLF4 and LASS2/TMSG1 was a target gene of KLF4 (22). In this experiment, when we interfered with ATP6V0C using siRNA, the expression of LASS2/TMSG1 was significantly decreased, perhaps due to activation of those transcriptional factors bound with the 
inhibitory regulatory region upstream of LASS2/TMSG1, thus inhibiting the expression of LASS2/TMSG1. Certainly, the precise molecular mechanism warrants further exploration.

In summary, silencing of ATP6V0C in PC-3M-1E8 cells inhibited V-ATPase activity, decreased extracellular hydrogen ion concentration and in turn decreased activation of secreted MMP-9, which was in accordance with the inhibition of cell migration and invasion in vitro, in addition to a marked decrease of LASS2/TMSG1 expression probably through positive feedback. Thus, we concluded that silencing of the ATP6V0C gene could effectively suppress migration and invasion of prostate cancer cells through the inhibition of the function of V-ATPase, however not through a LASS2/TMSG1-dependent manner. Therefore ATP6V0C inhibitors are promising therapeutic targets for advanced prostate cancer.

\section{Acknowledgements}

The present study was supported by the National Sciences Foundation of China (81572533). Thanks to Dr B.L. for data analysis.

\section{References}

1. Siegel R, Ma J, Zou Z and Jemal A: Cancer statistics, 2014. CA Cancer J Clin 64: 9-29, 2014.

2. Sennoune SR, Luo D and Martínez-Zaguilán R: Plasmalemmal vacuolar-type $\mathrm{H}^{+}$-ATPase in cancer biology. Cell Biochem Biophys 40: 185-206, 2004.

3. Cipriano DJ, Wang Y, Bond S, Hinton A, Jefferies KC, Qi J and Forgac M: Structure and regulation of the vacuolar ATPases. Biochim Biophys Acta 1777: 599-604, 2008.

4. Nishi $\mathrm{T}$ and Forgac M: The vacuolar $\left(\mathrm{H}^{+}\right)$-ATPases - nature's most versatile proton pumps. Nat Rev Mol Cell Biol 3: 94-103, 2002.

5. Pan H, Qin WX, Huo KK, Wan DF, Yu Y, Xu ZG, Hu QD, Gu KT, Zhou XM, Jiang HQ, et al: Cloning, mapping, and characterization of a human homologue of the yeast longevity assurance gene LAG1. Genomics 77: 58-64, 2001.

6. Yu W, Wang L, Wang Y, Xu X, Zou P, Gong M, Zheng J, You J, Wang H, Mei F, et al: A novel tumor metastasis suppressor gene LASS2/TMSG1 interacts with vacuolar ATPase through its homeodomain. J Cell Biochem 114: 570-583, 2013.

7. Liu Y, Zheng J, Fang W, You J, Wang J, Cui X and Wu B: Isolation and characterization of human prostate cancer cell subclones with different metastatic potential. Zhonghua Bing Li Xue Za Zhi 28: 361-364, 1999 (In Chinese).

8. Ma C, Liu Y, Zheng J, Fang W, You J, Wang J, Cui X and Wu B: Identification of tumor metastasis related gene TMSG-1 by mRNA differential display. Sci China C Life Sci 45: 553-560, 2002.
9. Laviad EL, Albee L, Pankova-Kholmyansky I, Epstein S, Park H, Merrill AH Jr and Futerman AH: Characterization of ceramide synthase 2: Tissue distribution, substrate specificity, and inhibition by sphingosine 1-phosphate. J Biol Chem 283: 5677-5684, 2008

10. $\mathrm{Xu} \mathrm{X,} \mathrm{You} \mathrm{J} \mathrm{and} \mathrm{Pei} \mathrm{F:} \mathrm{Silencing} \mathrm{of} \mathrm{a} \mathrm{novel} \mathrm{tumor} \mathrm{metastasis}$ suppressor gene LASS2/TMSG1 promotes invasion of prostate cancer cell in vitro through increase of vacuolar ATPase activity. J Cell Biochem 113: 2356-2363, 2012.

11. Forgac M: Structure and properties of the vacuolar $\left(\mathrm{H}^{+}\right)$-ATPases. J Biol Chem 274: 12951-12954, 1999.

12. Ohta T, Numata M, Yagishita H, Futagami F, Tsukioka Y, Kitagawa H, Kayahara M, Nagakawa T, Miyazaki I, Yamamoto M, et al: Expression of $16 \mathrm{kDa}$ proteolipid of vacuolar-type $\mathrm{H}(+)$-ATPase in human pancreatic cancer. Br J Cancer 73: 1511-1517, 1996.

13. Sennoune SR, Bakunts K, Martínez GM, Chua-Tuan JL, Kebir Y, Attaya MN and Martínez-Zaguilán R: Vacuolar $\mathrm{H}^{+}$-ATPase in human breast cancer cells with distinct metastatic potential: Distribution and functional activity. Am J Physiol Cell Physiol 286: C1443-C1452, 2004.

14. Novina CD and Sharp PA: The RNAi revolution. Nature 430: 161-164, 2004.

15. Lu X, Qin W, Li J, Tan N, Pan D, Zhang H, Xie L, Yao G, Shu H, Yao M, et al: The growth and metastasis of human hepatocellular carcinoma xenografts are inhibited by small interfering RNA targeting to the subunit ATP6L of proton pump. Cancer Res 65: 6843-6849, 2005.

16. Fei P, Junyu N, Jiangfeng Y, Jingpin Y, Yuping W, Zhihui H, Jieliang W, Xianglin C, Shaomin Y and Jie Z: Monoclonal antibodies against human tumor metastasis suppressor gene-1 (TMSG-1): Preparation, characterization, and application. Hybrid Hybridomics 23: 318-325, 2004.

17. Fan S, Niu Y, Tan N, Wu Z, Wang Y, You H, Ke R, Song J, Shen Q, Wang W, et al: LASS2 enhances chemosensitivity of breast cancer by counteracting acidic tumor microenvironment through inhibiting activity of V-ATPase proton pump. Oncogene. 32: $1682-1690,2013$

18. Xu XY, Pei F and You JF: TMSG-1 and its roles in tumor biology. Chin J Cancer 29: 697-702, 2010.

19. Xu X, Liu B, Zou P, Zhang Y, You J and Pei F: Silencing of LASS2/TMSG1 enhances invasion and metastasis capacity of prostate cancer cell. J Cell Biochem 115: 731-743, 2014.

20. Mei F, You J, Liu B, Zhang M, Liu J, Zhang B and Pei F: LASS2/TMSG1 inhibits growth and invasion of breast cancer cell in vitro through regulation of vacuolar ATPase activity. Tumour Biol 36: 2831-2844, 2015.

21. Gong M, Yu W, Pei F, You J, Cui X, McNutt MA, Li G and Zheng J: KLF6/Sp1 initiates transcription of the $t m s g-1$ gene in human prostate carcinoma cells: An exon involved mechanism. J Cell Biochem 113: 329-339, 2012.

22. Fan SH, Wang YY, Wu ZY, Zhang ZF, Lu J, Li MQ, Shan Q, Wu DM, Sun CH, Hu B, et al: AGPAT9 suppresses cell growth, invasion and metastasis by counteracting acidic tumor microenvironment through KLF4/LASS2/V-ATPase signaling pathway in breast cancer. Oncotarget 6: 18406-18417, 2015. 